\title{
A study of the constant mode in charmonium correlators at finite temperature
}

\section{Takashi Umeda*}

Graduate School of Pure and Applied Sciences, University of Tsukuba, Tsukuba, Ibaraki 305-8571, Japan

E-mail: tumeda@het.ph.tsukuba.ac.jp

Recent studies of the spectral functions of charmonia in lattice QCD suggest survival of the $J / \psi$ state in the deconfinement phase till relatively high temperature. Based on the studies, different scenarios of the $J / \psi$ suppression are discussed to understand experimental results in Heavy Ion Collision experiments. The scenarios require the information on the dissociation temperatures of $\chi_{c}$ and $\psi^{\prime}$ as well as that of $J / \psi$. In order to investigate these states in finite temperature lattice QCD, we have to consider an effect of the characteristic constant mode in meson correlators. As a result of the study of the constant mode, we find that most drastic changes in charmonium correlators for the $\chi_{c}$ states just above the deconfinement transition are caused by the constant mode. It may indicate the survival of the $\chi_{c}$ states after the deconfinement transition until, at least, $1.4 T_{c}$.

The XXV International Symposium on Lattice Field Theory

July 30-4 August 2007

Regensburg, Germany

\footnotetext{
* Speaker.
} 


\section{Introduction}

Study of heavy quarkona is important to understand the quark gluon plasma (QGP) formation in heavy ion collision experiments e.g. the RHIC experiment at Brookhaven National Laboratory. Recent studies of the spectral function of charmonium above $T_{c}$ suggest that hadronic excitations corresponding to the $J / \psi$ may survive in the deconfi nement phase till relatively high temperature [1-6]. Such results of the strongly interacting QGP may affect scenarios of the $J / \psi$ suppression $[7,8]$. Therefore the determination of the accurate dissociation temperature of $J / \psi$ is required for many phenomenological studies.

In order to study temporal correlators at fi nite temperature, one has to take account of the characteristic contribution caused by the fi nite temporal extent. Usually meson correlators are interpreted by the diagram with quark and anti-quark propagators like it is sketched in Fig. 1(a). However in the case of a system with a fi nite temporal extent, a wraparound (scattering) contribution, as shown in Fig. 1(b), has to be included as well. The effects are also known even at zero temperature cases e.g. the study of two pion correlators [9], and pentaquark correlators [10]. In the case, the contribution is usually removed using e.g. Dirichlet boundary conditions [10] or appropriate analyses [9]. On the other hand, at fi nite temperature, the latter contribution is genuine, and it provides a constant contribution to the correlator as a zero energy mode. Due to the contribution, correlators of such meson-like operators, e.g. charmonia, may be drastically changed just after the deconfi nement transition.

In this proceeding, we show how the constant mode affects the thermal effect in the charmonium correlators or the spectral functions. Details of the study has already been reported in the published paper [11].

\section{Constant mode in the free quark case}

\subsection{Meson-like correlators in the free quark case}

In order to investigate the constant contribution, we fi rst consider the free quark case of QCD, in which the constant contribution can be easily calculated. Here we defi ne the meson-like correlators with quark bilinear operators, $O_{\Gamma}(\vec{x}, t)=\bar{q}(\vec{x}, t) \Gamma q(\vec{x}, t)$, in the free quark case this gives for the correlator,

$$
C(t)=\sum_{\vec{x}}\left\langle O_{\Gamma}(\vec{x}, t) O_{\Gamma}^{\dagger}(\overrightarrow{0}, 0)\right\rangle,
$$

where $\Gamma$ are appropriate $4 \times 4$ matrices, i.e. $\gamma_{5}, \gamma_{i}, 1$, and $\gamma_{i} \gamma_{5}$ for pseudoscalar(Ps), vector $(\mathrm{V})$, scalar(S), and axialvector(Av) channels respectively. In this paper we calculate correlators for degenerate quark masses, constructed from bilinear operators, with vanishing spatial momentum as the simplest case, which is aiming at studies of charmonium at fi nite temperature discussed latter. The spectral function of the correlator is defi ned by

$$
\begin{aligned}
C(t)= & \int_{0}^{\infty} d \omega \rho_{\Gamma}(\omega) K(\omega, t), \\
& K(\omega, t)=\frac{\cosh \left(\omega\left(\frac{L_{t}}{2}-t\right)\right)}{\sinh \left(\omega \frac{L_{t}}{2}\right)},
\end{aligned}
$$

and has been calculated in Ref. $[12,13]$ in the high temperature limit. 


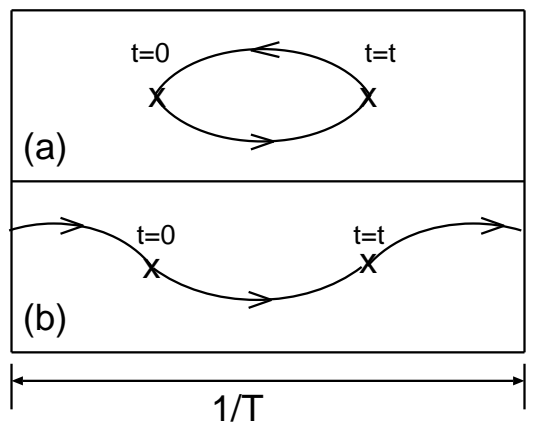

Figure 1: A sketch of quark line diagrams for a meson-like correlator in a system with finite temporal extent. Vertical lines show the boundaries in temporal direction.

\subsection{Free quark calculations on a lattice}

Here we present the numerical calculations of the meson-like correlators for the free quarks on a lattice. The calculations are performed on an isotropic $N_{s}^{3} \times N_{t}=16^{3} \times 32$ lattice. The free quark is described by the Wilson quark action with a bare quark mass of $m_{q}=0.2$.

Figure 2 (left panel) shows the effective masses of the correlators defi ned above. The effective mass is defi ned by the correlator at successive time slices.

$$
\frac{C(t)}{C(t+1)}=\frac{\cosh \left[m_{\mathrm{eff}}(t)\left(\frac{N_{t}}{2}-t\right)\right]}{\cosh \left[m_{\mathrm{eff}}(t)\left(\frac{N_{t}}{2}-t-1\right)\right]}
$$

The effective masses of the meson-like correlators with the free quarks should approach the energy of the two quark state without momentum for the S-wave states (Ps and V channels) and with a minimum momentum for the P-wave states ( $\mathrm{S}$ and $\mathrm{Av}$ channels) except for the zero energy mode. In the Ps and $\mathrm{V}$ channels, the zero relative momentum processes have no constant contribution and their effective masses approach the expected values. In the $S$ and Av channels, on the other hand, their effective masses approach the zero energy level rather than the energy of the two quark states.

\subsection{Analysis to avoid the constant contribution}

Next we consider how to estimate the effect of the constant mode. Of course the cosh + constant fi $t$ enables us to do so, but here we present the method without any fi $t$ analyses. The method uses the midpoint subtracted correlator,

$$
\bar{C}(t)=C(t)-C\left(N_{t} / 2\right) .
$$

One can also defi ne the effective mass of the subtracted correlator,

$$
\frac{\bar{C}(t)}{\bar{C}(t+1)}=\frac{\sinh ^{2}\left[\frac{1}{2} m_{\mathrm{eff}}^{\mathrm{sub}}(t)\left(\frac{N_{t}}{2}-t\right)\right]}{\sinh ^{2}\left[\frac{1}{2} m_{\mathrm{eff}}^{\mathrm{sub}}(t)\left(\frac{N_{t}}{2}-t-1\right)\right]} .
$$

Figure 2 (right panel) shows the effective mass $m_{\mathrm{eff}}^{\mathrm{sub}}(t)$, defi ned in Eq. (2.5), from the free quark results for the $N_{s}=16$. The effective masses are equivalent to the usual effective mass 


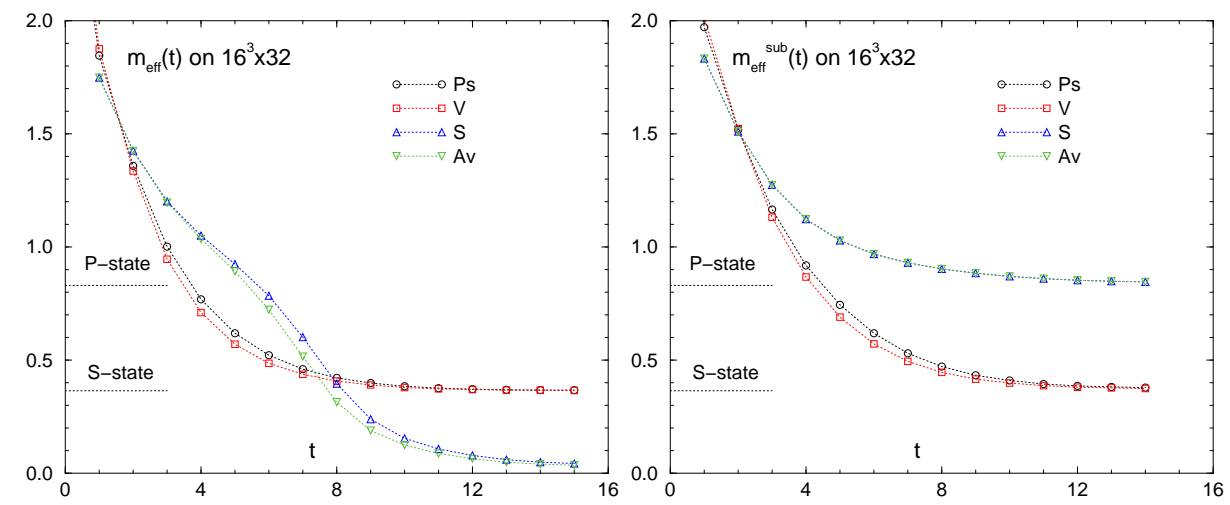

Figure 2: (Left) Effective masses of meson-like correlators for each channel. These are free quark calculations on a $16^{3} \times 32$ lattice.

shown in Fig. 2 (left panel) except for the effects of the constant mode. The expected energies of the lowest two quark states for the S-wave and the P-wave states are shown in both fi gures of Fig. 2. In contrast to the case of the usual effective masses, the subtracted effective masses $m_{\mathrm{eff}}^{\mathrm{sub}}(t)$ approach the expected values even in the P-wave states. The analysis to avoid the constant contribution works well at least in the free quark case.

The method using the midpoint subtracted correlators can also be applied to studies of spectral functions $\rho_{\Gamma}(\omega)$ by the modifi cation of the kernel,

$$
\begin{aligned}
\bar{C}(t)= & \int_{0}^{\infty} d \omega \rho_{\Gamma}(\omega) K^{\mathrm{sub}}(\omega, t), \\
& K^{\mathrm{sub}}(\omega, t)=\frac{2 \sinh ^{2}\left(\frac{\omega}{2}\left(\frac{N_{t}}{2}-t\right)\right)}{\sinh \left(\omega \frac{N_{t}}{2}\right)} .
\end{aligned}
$$

By using the alternative kernel $K^{\mathrm{sub}}(\omega, t)$, it is possible to extract the spectral function without the contribution in $\omega \ll T$ from e.g. the Maximum Entropy Method.

\section{Quenched QCD case at finite temperature}

\subsection{Lattice setup}

In this section we demonstrate the effect of the constant mode and the analysis to avoid it in quenched QCD at fi nite temperature. Here we calculate the charmonium correlators, which are defi ned like in Sect. 2.2 at the charm quark mass.

The gauge confi gurations have been generated by an standard plaquette gauge action with a lattice gauge coupling constant, $\beta=6.10$ and a bare anisotropy parameter $\gamma_{G}=3.2108$. The defi nition of the action and parameters are the same as that adopted in Ref. [14]. Lattice sizes are $20^{3} \times N_{t}$ where $N_{t}=160$ at $T=0$ and $N_{t}=32,26$ and 20 at $T>0$. The lattice spacings are $1 / a_{s}=2.030(13) \mathrm{GeV}$ and $1 / a_{t}=8.12(5) \mathrm{GeV}$ which are determined from the hadronic radius $r_{0}=0.5 \mathrm{fm}$. The physical volume size is approximately equal to $(2 \mathrm{fm})^{3}$ and the temperatures at $N_{t}=32,26$ and 20 are $T=0.88 T_{c}, 1.08 T_{c}$ and $1.40 T_{c}$ respectively. 


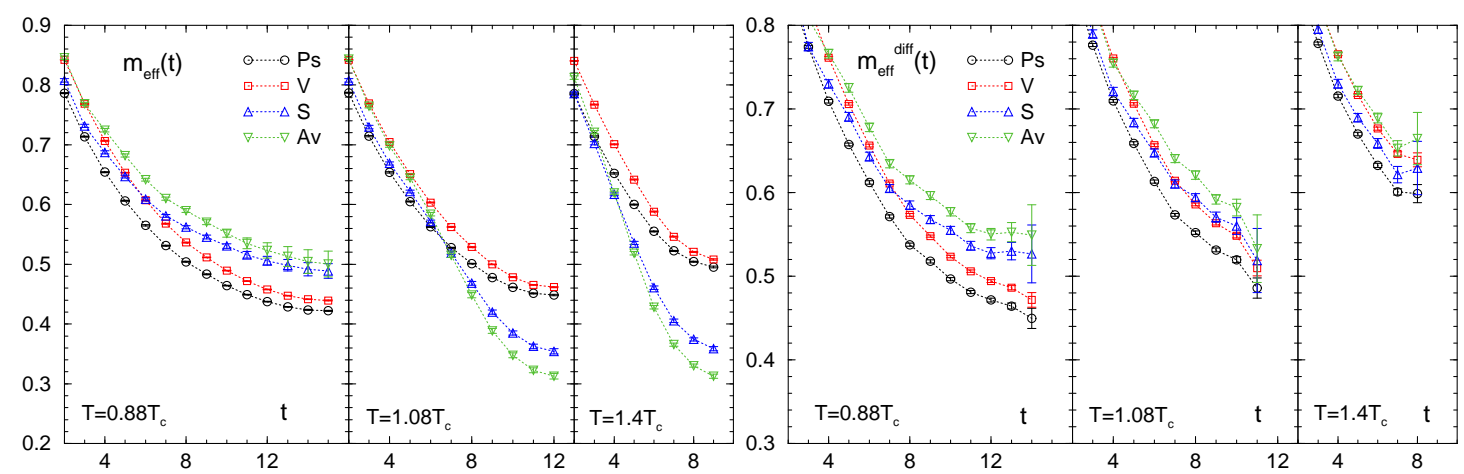

Figure 3: (Left) Effective masses from the usual charmonium correlators. (Right) Effective masses from the midpoint subtracted correlators. These are results with local operators for each channel in quenched QCD at finite temperature.

For the quark fi elds, we adopt an $O(a)$ improved Wilson quark action with tadpole improved tree level clover coeffi cients. Although the defi nition of the quark action is the same as in Ref. [14], we adopt the different choice of the Wilson parameter $r=1$ to suppress effects of lattice artifacts in higher states of the charmonium [12].

\subsection{Finite temperature results}

First we show the usual effective masses, $m_{\mathrm{eff}}(t)$ at fi nite temperature in Fig. 3 (Left panel). Since these are results obtained with local operators, there is no plateau region at any temperatures or channels we chose. It can be expected from the zero temperature calculation and the effective masses show reasonable behavior below $T_{c}$, at which the charmonium correlators does not show large difference from the zero temperature results $[2,3,6]$. Just above $T_{c}$, we see drastic changes in the P-wave state channels. The changes may cause the dissolution of $\chi_{c}$ states just above $T_{c}$ as already reported in Ref. $[3,5,6]$. On the other hand, the S-wave states, i.e. the Ps and V channels, show small changes up to $1.4 T_{c}$. This temperature dependences are shown in Fig. 3 (left panel) for each channel. When we compare the effective masses at the same time slices, we fi nd no large change up to $1.4 T_{c}$ in both channels as well. The results are consistent with previous lattice studies of the charmonium spectral functions [2-6], in which the spectral functions for the Ps and V channels in the deconfi nement phase (but at not so high temperature) show a peak structure around the $\eta_{c}$ and $J / \psi$ masses like the result at zero temperature.

\subsection{Midpoint subtracted correlator analysis}

Figure 3 (right panel) shows the results of $m_{\mathrm{eff}}^{\mathrm{sub}}(t)$ in quenched QCD at fi nite temperature. In the P-wave states, the drastic changes of the usual effective masses $m_{\text {eff }}(t)$ are absent in the effective masses from the midpoint subtracted correlators $m_{\mathrm{eff}}^{\text {sub }}(t)$. Furthermore the similar behaviors of the effective masses hold till $1.4 T_{c}$ as in the case of the S-wave states. The results indicate that the drastic changes of the usual correlators (and the effective masses) are caused only by the constant mode to the correlators. The situation is very similar to the free quark case discussed in Sect. 2. 


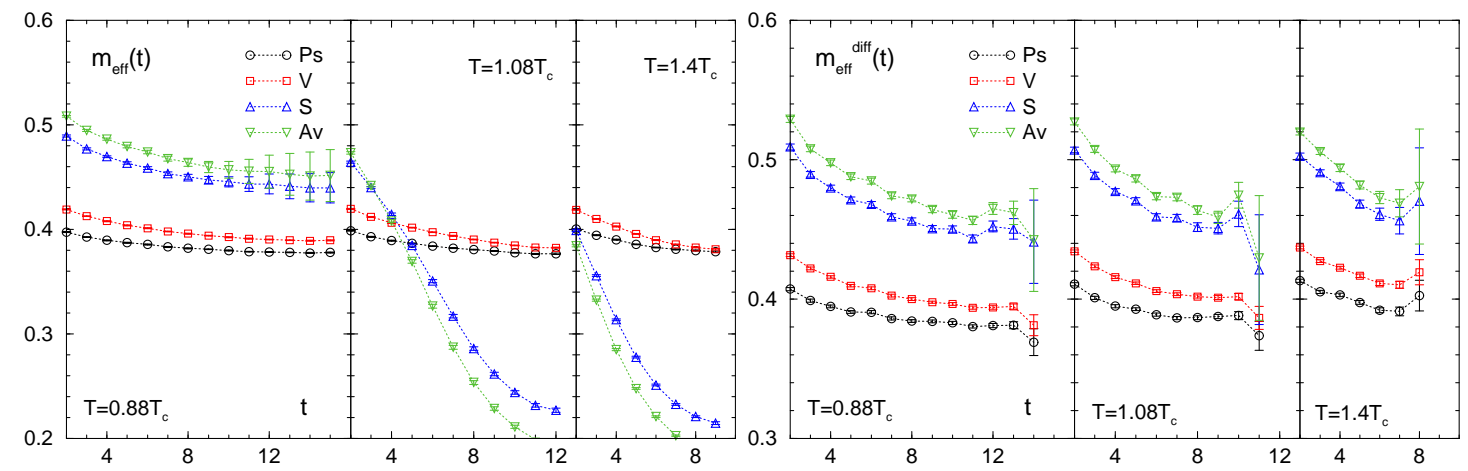

Figure 4: (Left) Effective masses from the usual charmonium correlators. (Right) Effective masses from the midpoint subtracted correlators. These are results with the spatially extended operators for each channel in quenched QCD at finite temperature.

\subsection{Results with spatially extended operators}

Finally we present the results of meson correlators with spatially extended operators. The spatially extended operators are constructed with a smearing function which yields a spatial distribution of the quark (and the anti-quark) source(s). The spatially extended operators are defi ned by $O_{\Gamma}(\vec{x}, t)=\sum_{\vec{y}} \phi(\vec{y}) \bar{q}(\vec{x}-\vec{y}, t) \Gamma q(\vec{x}, t)$ with the smearing function $\phi(\vec{x})$ in Coulomb gauge. In this calculation the spatially extended operators are adopted only in the source operators, the sink operators are local in any cases. The smearing function is the same as that in Ref. [2], i.e. $\phi(\vec{x})=\exp \left(-A|\vec{x}|^{P}\right)$ where $A$ and $P$ are parameters determined by the matching with the charmonium wave function as $A=0.2275$ and $P=1.258$. Figure 4 (left panel) is the result for the usual effective masses with the spatially extended operators. Other conditions are the same as in Fig. 3. Below $T_{c}$, in contrast to the case of local operators the effective masses reach a plateau due to the larger overlap with the lowest state. Above $T_{c}$ the effective masses of the P-wave states change more than in the case of local operators. Because the constant contribution is enhanced by the smearing function more than the other contributions in the case. Figure 4 (right panel) is the same fi gure as Fig. 3, but with spatially extended operators. We fi nd similar behavior for both cases of operators.

\section{Conclusion}

Let us discuss details on $\chi_{c}$ states using the results of the previous sections. In this study we find that the drastic changes of the (usual) correlators in the P-wave states are caused by the constant contribution, while the correlators without the constant mode yield small changes till, at least, $1.4 T_{c}$ even in the $\mathrm{P}$-wave states. The changes are of similar size as that of the $\mathrm{S}$-wave states as one can see in Fig. 3 and Fig. 4.

Although the constant mode coming from the scattering process is important to investigate some transport phenomena, it does not affect the spectral function in the $\omega \gg T$ region for the charmonium correlators with the zero spatial momentum. Therefore the dissolution of the $\chi_{c}$ states just above $T_{c}$ as discussed in e.g. Ref. $[3,5,6]$ might be misleading. When there is the constant contribution, the hadronic state is no longer the lowest state. The situation provides some diffi cul- 
ties in the analysis to reconstruct the spectral function in the higher energy part $\omega \gg T$ even if the MEM is applied [15].

In principle the MEM analysis can extract the correct spectral function even if the constant contribution exists, however it is diffi cult to reproduce the non-lowest part of the spectral function correctly by the MEM analysis at fi nite temperature with typical statistics. In order to investigate the properties of the $\chi_{c}$ states above $T_{c}$, a careful analysis taking the constant contribution into account is necessary. A correct MEM analysis of the correlator with and without the constant contribution should give the same results, up to the low energy region $\omega \ll T$. Such a comparison can be easily performed by the MEM analysis with the alternative kernel of Eq. (2.7) for the midpoint subtracted correlators. Failure to do so signals unreliable MEM results.

\section{Acknowledgments}

The simulations have been performed on supercomputers (NEC SX-5) at the Research Center for Nuclear Physics (RCNP) at Osaka University and (NEC SX-8) at the Yukawa Institute for Theoretical Physics (YITP) at Kyoto University. This work has been authored under the contract number DE-AC02-98CH1-886 with the U.S. DOE and Nos.19549001 with Grants-in-Aid of the Japanese MEXT.

\section{References}

[1] T. Umeda, R. Katayama, O. Miyamura and H. Matsufuru, Int. J. Mod. Phys. A 16 (2001) 2215 [hep-lat/0011085].

[2] T. Umeda, K. Nomura and H. Matsufuru, Eur. Phys. J. C 39S1 (2005) 9 [hep-lat/ 0211003 ].

[3] S. Datta, F. Karsch, P. Petreczky and I. Wetzorke, Phys. Rev. D 69 (2004) 094507 [hep-lat/0312037].

[4] M. Asakawa and T. Hatsuda, Phys. Rev. Lett. 92 (2004) 012001 [hep-lat/ 0308034 ].

[5] G. Aarts, C. R. Allton, R. Morrin, A. P. O. Cais, M. B. Oktay, M. J. Peardon and J. I. Skullerud, POS (LATTICE2006) 126 [hep-lat/0610065].

[6] A. Jakovac, P. Petreczky, K. Petrov and A. Velytsky, hep-lat/0611017.

[7] T. Hashimoto, K. Hirose, T. Kanki and O. Miyamura, Phys. Rev. Lett. 57 (1986) 2123.

[8] T. Matsui and H. Satz, Phys. Lett. B 178 (1986) 416.

[9] C. Kim, Nucl. Phys. Proc. Suppl. 129 (2004) 197 [hep-lat/0311003].

[10] T. T. Takahashi, T. Umeda, T. Onogi and T. Kunihiro, Phys. Rev. D 71 (2005) 114509 [hep-lat/0503019].

[11] T. Umeda, Phys. Rev. D 75 (2007) 094502 [hep-lat/ 0701005$].$

[12] F. Karsch, E. Laermann, P. Petreczky and S. Stickan, Phys. Rev. D 68 (2003) 014504 [hep-lat/0303017].

[13] G. Aarts and J. M. Martinez Resco, Nucl. Phys. B 726 (2005) 93 [hep-lat/ 050700 4].

[14] H. Matsufuru, T. Onogi and T. Umeda, Phys. Rev. D 64 (2001) 114503 [hep-lat/ 0107001 ].

[15] T. Umeda and H. Matsufuru, PoS (LAT2005) 154 [hep-lat/0510026]. 